PROCEEDINGS OF THE

AMERICAN MATHEMATICAL SOCIETY

Volume 125, Number 11, November 1997, Pages 3339-3345

S 0002-9939(97)03979-8

\title{
A LOGARITHMIC SOBOLEV INEQUALITY ON THE REAL LINE
}

\author{
J. MICHAEL PEARSON
}

(Communicated by Christopher D. Sogge)

\begin{abstract}
A new logarithmic Sobolev inequality for the real line is obtained. The inequality is obtained by applying a differentiation argument to a sharp Sobolev inequality due to Nagy, and is $L^{p}$ rather that $L^{2}$ in structure.
\end{abstract}

\section{INTRODUCTION}

Logarithmic Sobolev inequalities have played a central role in the study of norm estimates for diffusion semigroups. The connection between logarithmic inequalities and diffusion semigroups was first recognized by Gross [6], who proved a logarithmic Sobolev inequality for gaussian measure corresponding to Nelson's [10] hypercontractive estimate for the Hermite semigroup (see section 3.1 below). Similar estimates have been obtained for diffusion semigroups on a variety of probability spaces, including the heat semigroup (Mueller and Weissler [8]) and the Poisson semigroup (Beckner [3]) on the $n$-sphere $S^{n}$. Results describing necessary conditions for hypercontractivity on probability spaces were given in terms of multipliers on orthogonal polynomials by Janson [7], while sufficient conditions for hypercontractivity in terms of the generator of the diffusion semigroup were obtained by Bakry and Emery [1].

In the examples mentioned above, logarithmic Sobolev inequalities can be viewed as infinitesimal versions of the corresponding hypercontractive estimates. This was the point of view of Gross [6]. In other cases it is possible to obtain logarithmic inequalities as limiting cases (differentiated forms) of a Sobolev inequality. This idea played a central role in Beckner's work [3] on the Poisson semigroup. This point of view provides the motivation for the present work, where we will apply a differentiation argument to an inequality due to Nagy [9] to obtain a new logarithmic inequality on the real line (inequality (2.3)).

While the argument used to obtain inequality (2.3) is similar to the arguments used in the works cited above, the structure of the inequality is significantly different. This is the first logarithmic inequality known to the author where the estimate is $L^{p}$ rather than $L^{2}$.

Received by the editors March 19, 1996 and, in revised form, June 14, 1996.

1991 Mathematics Subject Classification. Primary 42A99; Secondary 46 E35.

(C)1997 American Mathematical Society 


\section{THE LOGARITHMIC INEQUALITY}

Let

$$
J_{q}=J_{q}(f)=\int_{-\infty}^{\infty}|f(x)|^{q} d x \text { and } K_{p}=K_{p}(f)=\int_{-\infty}^{\infty}\left|f^{\prime}(x)\right|^{p} d x .
$$

The following inequality is due to Nagy [9].

Theorem 2.1 (Nagy). For $q, \beta>0$ and $p \geq 1$,

$$
J_{q+\beta} \leq\left(\frac{s}{2} H\left(\frac{s}{\beta}, \frac{p-1}{p}\right)\right)^{\frac{\beta}{s}} J_{q}^{1+\beta \frac{p-1}{p s}} K_{p}^{\frac{\beta}{p s}},
$$

where $s=1+\frac{p-1}{p} q$ and

$$
H(u, v)=\frac{(u+v)^{-(u+v)} \Gamma(1+u+v)}{u^{-u} v^{-v} \Gamma(1+u) \Gamma(1+v)}, H(u, 0)=H(0, v)=1 .
$$

For $p>1$, extremals are of the form $f(t)=a g(|b t+c|)$ where $u=g(t), t \geq 0$, is the inverse function of

$$
t=\int_{u}^{1} \frac{d s}{\left[s^{q}\left(1-s^{\beta}\right)\right]^{1 / p}}, 0 \leq u \leq 1 .
$$

If $q \geq p$, set $g(t)>0$ for all $t>0$. If $q<p$, set $g(t)=0$ for all $t \geq t_{0}$, where

$$
t_{0}=\int_{0}^{1} \frac{d s}{\left[s^{q}\left(1-s^{\beta}\right)\right]^{1 / p}} .
$$

For a function $f: \mathbb{R} \rightarrow \mathbb{R}$ (or $\mathbb{C}$ ), we define the symmetric decreasing rearrangement $\tilde{f}$ of $f$ by

$$
\begin{aligned}
& \tilde{f} \text { is non-negative and even, } \\
& |s|>|t| \Rightarrow \widetilde{f}(s) \leq \widetilde{f}(t), \text { and } \\
& m\{|f|>y\}=m\{\widetilde{f}>y\} .
\end{aligned}
$$

By definition, we will have $J_{q}(f)=J_{q}(\tilde{f})$, while standard results on rearrangements yield $K_{p}(f) \leq K_{p}(\widetilde{f})$. Without loss of generality, then, we can restrict attention to this class of functions when considering Nagy's result (inequality (2.1)). We refer the reader to Nagy's work [9] for a proof of the theorem. However, for $p=1$, it is easy to see that strict inequality holds in (2.1).

For $p=1$ and $f=\tilde{f} \in L^{1} \cap L^{\infty}$ smooth, we will have

$$
\begin{aligned}
K_{1} & =\int_{-\infty}^{\infty}\left|f^{\prime}(t)\right| d t \\
& \geq-2 \int_{0}^{a} f^{\prime}(t) d t=2(f(0)-f(a)) \\
& \rightarrow 2 f(0), a \rightarrow \infty,
\end{aligned}
$$

or

$$
f(0) \leq \frac{1}{2} K_{1}
$$


so that, in general, we will have

$$
\|f\|_{\infty} \leq \frac{1}{2} K_{1}
$$

Using this we find

$$
\begin{aligned}
J_{q+\beta} & =\int_{-\infty}^{\infty}(f(t))^{q+\beta} d t \\
& <\|f\|_{\infty}^{\beta} J_{q} \\
& \leq\left(\frac{1}{2} K_{1}\right)^{\beta} J_{q} .
\end{aligned}
$$

This is Nagy's result for $p=1$.

Using Nagy's result we can show that the following logarithmic Sobolev inequality holds for functions on the real line.

Theorem 2.2. For $q>0, p \geq 1$, and $s=1+\frac{p-1}{p} q$,

$$
\begin{aligned}
& \int_{-\infty}^{\infty}|f(x)|^{q} \ln |f(x)| d x \\
& \quad \leq J_{q}\left(\frac{1}{s} \ln \left(\frac{s\left(\frac{p-1}{p}\right)^{\frac{p-1}{p}}}{2 \Gamma\left(2-\frac{1}{p}\right)}\right)-\frac{p-1}{p s}+\frac{p-1}{p s} \ln \left(J_{q}\right)+\frac{1}{p s} \ln \left(K_{p}\right)\right) .
\end{aligned}
$$

For $p>1$, extremals are of the form $f(t)=a h(|b t+c|)$ where $u=h(t), t \geq 0$, is the inverse function of

$$
t=\int_{0}^{\ln (1 / u)} w^{-1 / p} e^{-w(1-q / p)} d w, 0<u \leq 1
$$

If $q \geq p$, then $h(t)>0$ for all $t>0$. If $q<p$, then $h(t)=0$ for all $t \geq t_{0}$, where

$$
t_{0}=\left(\frac{p}{p-q}\right)^{(p-1) / p} \Gamma\left(\frac{p-1}{p}\right) \text {. }
$$

Proof. To prove inequality (2.3), we will apply a differentiation argument to (2.1).

Note that, as $\beta$ decreases to 0 , inequality (2.1) becomes an identity since

$$
\lim _{\beta \rightarrow 0^{+}}\left(\frac{s}{2} H\left(\frac{s}{\beta}, \frac{p-1}{p}\right)\right)^{\frac{\beta}{s}}=1
$$

independent of $p, s \geq 1$. Subtracting $J_{q}$ and dividing by $\beta>0$ on both sides of inequality (2.1) yields

$$
\frac{J_{q+\beta}-J_{q}}{\beta} \leq J_{q}\left(\frac{\left(\frac{s}{2} H\left(\frac{s}{\beta}, \frac{p-1}{p}\right)\right)^{\frac{\beta}{s}} J_{q}^{\beta \frac{p-1}{p s}} K_{p}^{\frac{\beta}{p s}}-1}{\beta}\right)
$$

Inequality (2.5) expresses a relationship between difference quotients. The proof of Theorem 2.2 now consists of evaluating the limit as $\beta$ decreases to 0 in (2.5). We easily find

$$
\lim _{\beta \rightarrow 0^{+}}\left(\frac{J_{q+\beta}-J_{q}}{\beta}\right)=\frac{d}{d q}\left(\int_{-\infty}^{\infty}|f(x)|^{q} d x\right)=\int_{-\infty}^{\infty}|f(x)|^{q} \ln |f(x)| d x .
$$


In order to evaluate the desired limit of the right hand side of (2.5), we use the asymptotic expansion ([5], vol. 1, 1.18(4), p. 47)

$$
\frac{\Gamma(z+a)}{\Gamma(z)}=t^{a}\left(1+\frac{1}{2 z}+O\left(z^{-2}\right)\right)
$$

together with the binomial theorem to find

$$
\left(\frac{\Gamma\left(\frac{s}{\beta}+\frac{p-1}{p}\right)}{\Gamma\left(\frac{s}{\beta}\right)}\right)^{\frac{\beta}{s}}=\left(\frac{s}{\beta}\right)^{\frac{\beta}{s}\left(\frac{p-1}{p}\right)}+O\left(\beta^{2-\beta \frac{p-1}{p s}}\right) .
$$

Using this together with elementary properties of the gamma function, we obtain

$$
\begin{aligned}
\left(\frac{s}{2} H\left(\frac{s}{\beta}, \frac{p-1}{p}\right)\right)^{\frac{\beta}{s}}= & \left(\frac{s\left(\frac{p-1}{p}\right)^{\frac{p-1}{p}}}{2 \Gamma\left(2-\frac{1}{p}\right)}\right)^{\frac{\beta}{s}}\left(\frac{1}{1+\beta\left(\frac{p-1}{p s}\right)}\right)^{1-\frac{\beta}{p s}} \\
& \times\left(\frac{s}{\beta}\right)^{-\frac{\beta}{s}\left(\frac{p-1}{p}\right)}\left(\frac{\Gamma\left(\frac{s}{\beta}+\frac{p-1}{p}\right)}{\Gamma\left(\frac{s}{\beta}\right)}\right)^{\frac{\beta}{s}} \\
= & \left(\frac{s\left(\frac{p-1}{p}\right)^{\frac{p-1}{p}}}{2 \Gamma\left(2-\frac{1}{p}\right)}\right)^{\frac{\beta}{s}}\left(\frac{1}{1+\beta\left(\frac{p-1}{p s}\right)}\right)^{1-\frac{\beta}{p s}}+O\left(\beta^{2}\right) \\
\equiv & C(p, s, \beta)+O\left(\beta^{2}\right) .
\end{aligned}
$$

From this estimate, we learn that

$$
\begin{array}{r}
\lim _{\beta \rightarrow 0^{+}} J_{q}\left(\frac{\left(\frac{s}{2} H\left(\frac{s}{\beta}, \frac{p-1}{p}\right)\right)^{\frac{\beta}{s}} J_{q}^{\beta \frac{p-1}{p s}} K_{p}^{\frac{\beta}{p s}}-1}{\beta}\right) \\
=\lim _{\beta \rightarrow 0^{+}} J_{q}\left(\frac{C(p, s, \beta) J_{q}^{\beta \frac{p-1}{p s}} K_{p}^{\frac{\beta}{p s}}-1}{\beta}\right) \\
=J_{q} \lim _{\beta \rightarrow 0^{+}}\left[\frac{d}{d \beta}\left(C(p, s, \beta) J_{q}^{\beta \frac{p-1}{p s}} K_{p}^{\frac{\beta}{p s}}\right)\right] .
\end{array}
$$

We can now use the definition of $C(p, s, b)$ given above and readily proceed to obtain inequality (2.3) as desired.

To see that the extremals satisfy the relation described in the statement of the theorem, we will use the dilation invariance of inequality (2.1). For fixed $p$, consider the function $f(t)=g\left(\beta^{1 / p}|t|\right)$, where $g$ is the function which defines extremals for Nagy's inequality (see relation (2.2) and the surrounding discussion) and observe that

$$
\lim _{\beta \rightarrow 0} \frac{1-s^{\beta}}{\beta \ln (1 / s)}=1
$$


Substituting into the relation (2.2), we see that $f$ will be an even function given for $t \geq 0$ as the inverse of the function

$$
\begin{aligned}
t & =\beta^{1 / p} \int_{u}^{1} \frac{d s}{\left[s^{q}\left(1-s^{\beta}\right)\right]^{1 / p}} \\
& \rightarrow \int_{u}^{1} \frac{d s}{\left(s^{q} \ln (1 / s)\right)^{1 / p}}, \text { as } \beta \rightarrow 0,0<u \leq 1
\end{aligned}
$$

Now make the substitution $w=\ln (1 / s)$ to obtain

$$
\lim _{\beta \rightarrow 0^{+}} t=\int_{0}^{\ln (1 / u)} w^{-1 / p} e^{-w(1-q / p)} d w .
$$

(The cases $q<p$ and $q \geq p$ are handled the same as in Nagy's theorem.) Since, for this choice of $f$ and for each $\beta>0$, inequality (2.5) is in fact an equality, and for $p>1$ the integral (2.4) converges for $0<u \leq 1$, we see that extremals for (2.3) are as claimed. For $p>1$ and $0<q<p$, we can express (2.4) as

$$
t=\left(\frac{p}{p-q}\right)^{(p-1) / p}\left[\Gamma\left(\frac{p-1}{p}\right)-\Gamma\left(\frac{p-1}{p}, \frac{p-q}{p} \ln (1 / u)\right)\right]
$$

where the incomplete gamma function $\Gamma(a, x)$ is given by

$$
\Gamma(a, x)=\int_{x}^{\infty} e^{-t} t^{a-1} d t, a>0, x \geq 0 .
$$

In particular, in this case we can determine that the extremal $f$ vanishes for

$$
|t| \geq t_{0}=\left(\frac{p}{p-q}\right)^{(p-1) / p} \Gamma\left(\frac{p-1}{p}\right) .
$$

In general, a closed form for the extremals cannot be found. However, for $1<$ $q=p,(2.4)$ yields

$$
t=\frac{p}{p-1}[\ln (1 / u)]^{(p-1) / p}
$$

which allows us to construct the extremal function $f$ explicitly:

$$
f(t)=\exp \left(-\left[\left(\frac{p-1}{p}\right)|t|\right]^{\frac{p}{p-1}}\right) .
$$

In this case, we can verify equality in (2.3) directly.

\section{Further REMARKS}

3.1. Gross' logarithmic Sobolev inequality. In previous work [11], we used a change of variables together with a differentiation argument applied to a sharp Sobolev inequality on the real line to give an elementary argument for deriving Gross' logarithmic Sobolev inequality

$$
\int_{-\infty}^{\infty}|g(t)|^{2} \ln |g(t)| d \mu(t) \leq \int_{-\infty}^{\infty}\left|g^{\prime}(t)\right|^{2} d \mu(t)
$$


for $\int_{-\infty}^{\infty}|g(t)|^{2} d \mu(t)=1$ and $d \mu(t)=(2 \pi)^{-1 / 2} e^{-t^{2} / 2} d t$ normalized gaussian measure. The product structure of gaussian measure allows the reduction of the $n$ dimensional problem to dimension one, so that as Gross [6] showed (3.1) is equivalent to Nelson's [10] hypercontractive estimate for the Hermite semigroup: for $1<p<q<\infty$,

$$
e^{-t} \leq \sqrt{\frac{p-1}{q-1}} \Rightarrow\left(\int_{\mathbf{R}^{n}}\left|e^{-t N} f\right|^{q} d \mu\right)^{1 / q} \leq\left(\int_{\mathbf{R}^{n}}|f|^{p} d \mu\right)^{1 / p}
$$

where $N=-\Delta+x \cdot \nabla$ and $d \mu=d \mu(x)=(2 \pi)^{-n / 2} e^{-|x|^{2} / 2} d x$.

3.2. Sharp estimates for Young's inequality and the heat semigroup on $\mathbb{R}^{n}$. If $f \in L^{p}\left(\mathbb{R}^{n}\right)$ and $g \in L^{q}\left(\mathbb{R}^{n}\right)$, the sharp form of Young's inequality for convolution obtained by Beckner [2] states that, for $1 \leq p, q, r \leq \infty$ and $1 / r=$ $1 / p+1 / q-1$,

$$
\|f * g\|_{r} \leq\left(A_{p} A_{q} A_{r}\right)^{n}\|f\|_{p}\|g\|_{q}
$$

where we write $\|\cdot\|_{p}=\left(\int_{\mathbb{R}^{n}}|\cdot|^{p} d x\right)^{1 / p}$ and $A_{p}=\left[p^{1 / p}\left(p^{\prime}\right)^{-1 / p^{\prime}}\right]^{1 / 2}$. In the same work, Beckner pointed out that Nelson's [10] hypercontractive estimate for the Hermite semigroup is equivalent to (3.3).

The heat diffusion semigroup on $\mathbb{R}^{n}$ is given by

$$
\left(e^{t \Delta} f\right)(x)=\int_{\mathbb{R}^{n}} \phi(t, x-y) f(y) d y,
$$

where

$$
\phi(t, x)=(4 \pi t)^{-n / 2} \exp \left(-|x|^{2} / 4 t\right) .
$$

From Beckner's estimate (3.3), it follows that the best possible norm estimates for this semigroup are given by

$$
\left\|e^{t \Delta} f\right\|_{q} \leq\left[A_{q} A_{p^{\prime}}\right]^{n}(4 \pi t r)^{-n / 2 r}\|f\|_{p}
$$

where $1<p \leq q<\infty, t>0, p^{-1}+\left(p^{\prime}\right)^{-1}=1$, and $r^{-1}=p^{-1}-q^{-1}$.

Applying a differentiation argument to (3.4), Weissler [13] obtained the logarithmic inequality

$$
\int_{\mathbb{R}^{n}} f^{2} \ln |f| d x \leq \frac{n}{4} \ln \left(\frac{2}{\pi n e} \int_{\mathbb{R}^{n}}|\nabla f|^{2} d x\right)
$$

valid for $\|f\|_{2}=1$. Weissler showed that (3.5) is equivalent to (3.4).

More recently, inequality (3.5) appeared in the work of Beckner [4]. Beckner used a change of variables and a variational argument to show that Gross' logarithmic Sobolev inequality (3.1) is equivalent to (3.5) and pointed out that inequality (3.5) for dimension $n=1$ could be derived from (2.1). In inequality (2.3), if we take $p=q=2$ (so that $s=2$ also), require $J_{2}=1$ and use the fact that $\Gamma(3 / 2)=\sqrt{\pi} / 2$, then we recover inequality (3.5) for $n=1$.

3.3. Other results. Weissler [13] compared the logarithmic inequality (3.5) to the Sobolev-Nirenberg inequalities

$$
\|\phi\|_{r} \leq C(n, r)\|\nabla \phi\|_{2}^{a}\|\phi\|_{2}^{1-a}
$$

where $r>2$ and $a=n(1 / 2-1 / r)$, and concluded that inequality (3.6) is, in some sense, stronger than (3.5). The structure of (3.6) is the same as Nagy's inequality (2.1), suggesting the possibility (in the special case $p=q=2$ ) of an argument 
analogous to the one used here for dimension $n>1$. Weinstein [12] has shown that extremals for (3.6) correspond to the ground state of a non-linear Schrödinger equation and developed a method to approximate the best constant to any desired accuracy. However, for dimension $n>1$, there is no known closed form expression for the constant $C(n, r)$.

\section{REFERENCES}

1. D. Bakry and M. Emery, 'Hypercontactivite de semi-grouptes de diffusion', C. R. Acad. Sc. Paris 299 (1984) 775-778. MR 86f:60097

2. W. Beckner, 'Inequalities in Fourier analysis', Ann. of Math. (2) 102 (1975), 159-182. MR 52:6317

3. W. Beckner, 'Sobolev inequalities, the Poisson semigroup and analysis on the sphere $S^{n}$, Proc. Nat. Acad. Sci. 89 (1992) 4816-4819. MR 93d:26018

4. W. Beckner, 'Pitt's inequality and the uncertainty principle', Proc. Amer. Math. Soc. 123 (1995) 1897-1905. MR 95g:42021

5. A. Erdelyi, et al, Higher Transcendental Functions, Vol I (McGraw-Hill, New York, 1953). MR 15:419i

6. L. Gross, 'Logarithmic Sobolev inequalities', Amer. J. Math. 97 (1975) 1061-1083. MR 54: 8263

7. S. Janson, 'On hypercontractivity for multipliers on orthogonal polynomials', Ark. Mat. 21 (1983) 97-110. MR 85g:47070

8. C. W. Mueller and F. B. Weissler, 'Hypercontractivity for the heat semigroup for ultraspherical polynomials on the $n$-sphere', J. Funct. Anal. 48 (1982) 252-283. MR 83m:47036

9. B. V. Sz. Nagy, 'Uber Integralungleichunger zwischen einer Funktion und ihrer Ableitung', Acta Sci. Math. (Szeged) 10 (1941) 64-74.

10. E. Nelson, 'The free Markov field', J. Funct. Anal. 12 (1973) 211-227. MR 49:8556

11. J. M. Pearson, 'An elementary proof of Gross' logarithmic Sobolev inequality', Bull. London Math. Soc. 25 (1993) 463-466. MR 94g:26028

12. M. I. Weinstein, 'Nonlinear Schrodinger equations and sharp interpolation estimates', Comm. Math. Physics 87 (1982/83) 567-576. MR 84d:35140

13. F. B. Weissler, 'Logarithmic Sobolev inequalities for the heat diffusion semigroup', Trans. Amer. Math. Soc. 237 (1978) 255-269. MR 80b:47057

Department of Mathematics and Statistics, Mississippi State University, Mississippi State, Mississippi 39762

E-mail address: pearson@math.msstate.edu 